\title{
Abundances for a Large Sample of Galactic Planetary Nebulae
}

\author{
V.V. Golovaty ${ }^{1}$ and Yu.F. Malkov ${ }^{2}$ \\ ${ }^{1}$ Astronomical Observatory of Lviv State University, Lviv, Ukraine; \\ ${ }^{2}$ Crimean Astrophysical Observatory, Nauchny, Crimea, Ukraine
}

We present a database on the chemical composition of 200 planetary nebulae (PNe) in our Galaxy. Abundances of He, C, N, O, Ne, Mg, Si, S, and Ar are found for 175, 64, 190, 200, $170,13,40,173$, and 187 PNe respectively. The abundances for all PNe are obtained with the same atomic data and by the same method of abundance determination, so the database is highly homogeneous. The method of the determination of nebular abundances based on a photoionization model grid is described by Golovaty et al. (1993). Observational data have been compiled from 105 papers published in 1976-1994. For most of PNe in our sample, the distances and central star masses are determined as well (Malkov, this volume).

Relations between the nebular abundances and abundance ratios are found to be in general agreement with recent theoretical predictions. Surprisingly, the calculations made for the evolution of stars in the Large Magellanic Cloud (Groenewegen and de Jong, 1994) show better agreement with our data than the calculations for stars in our Galaxy (Groenewegen et al., 1995). The correlation between the central star mass and the N/O ratio is clearly pronounced. There are no strong abundance anomalies in the Galactic bulge nebulae as compared with the Galactic disk PNe.

The abundances in Type II PNe together with those in blue compact galaxies and galactic H II regions (Chiappini and Maciel, 1994) were used to redetermine the pregalactic helium abundance $Y_{p}$ and the helium-to-metals enrichment ratio $d Y / d Z$. The resulting values are $Y_{p}=0.233$ and $d Y / d Z=4.30$.

The radial and vertical abundance gradients in the Galactic disk are evident for oxygen, neon, sulphur and argon, but their values $(-0.035 \mathrm{dex} / \mathrm{kpc}$ and $-0.15 \mathrm{dex} / \mathrm{kpc}$ respectively) are somewhat lower than found by other authors. We suppose it can be explained by different distance scales. An evolution of abundance gradients is suggested by our data: the nebulae with central star masses less than $\sim 0.60 \mathrm{M}_{\odot}$ (i.e., the nebulae formed by lowmassive, old progenitor stars) show the vertical gradient only but no radial one while both radial and vertical gradients are traced by the PNe with more massive central stars.

\section{REFERENCES}

Chiappini, C., and Maciel, W.J., 1994, Astron. Astrophys., 288, 921.

Golovaty, V.V., Dmiterko, V.I., Malkov, Yu.F., and Rokach, O.V., 1993, Astron. Zhurn., 70, 691.

Groenewegen, M.A.T., and de Jong, T., 1994, Astron. Astrophys., 282, 127.

Groenewegen, M.A.T., van den Hoek, L.B., and de Jong, T., 1995, Astron. Astrophys., 293, 381. 